\title{
A New Movement Authority Based on Vehicle-Centric Communication
}

\author{
Tuo Shen ${ }^{1}{ }^{1}$ and Haifeng Song $\mathbb{D D}^{2}$ \\ ${ }^{1}$ School of Optical-Electrical and Computer Engineering, University of Shanghai for Science and Technology, Shanghai 200093, China \\ ${ }^{2}$ Institute for Traffic Safety and Automation Engineering, Technische Universität Braunschweig, 38108 Braunschweig, Germany
}

Correspondence should be addressed to Haifeng Song; h.song@tu-braunschweig.de

Received 7 December 2017; Revised 16 February 2018; Accepted 4 March 2018; Published 4 April 2018

Academic Editor: Li Zhu

Copyright (C) 2018 Tuo Shen and Haifeng Song. This is an open access article distributed under the Creative Commons Attribution License, which permits unrestricted use, distribution, and reproduction in any medium, provided the original work is properly cited.

\begin{abstract}
The communication system that is presently applied in the European Train Control System can only support data exchange between vehicles and ground, but the direct vehicle-to-vehicle communication is not available. The details of interlocking information and other vehicles' movements are invisible to drivers who are the last defense to prevent unsafe scenarios. As connected vehicles have been envisioned to enhance transportation efficiency and improve safety, the direct vehicle-to-vehicle communication network is involved in this paper to increase the safety of railway transport. In this paper, a new train movement authority (MA+) is proposed. Apart from a wireless communication unit, this system does not require any other infrastructure. With the assistance of vehiclecentric communication technology, MA+ can detect the condition of switches and trains within a certain scope. In this paper, the system structure of MA+ is proposed. Additionally, different implementation scenarios are also discussed. The detection range is estimated and validated based on mathematical calculation and experimental equations. An application demo of MA+ is presented on the Driver Machine Interface of the onboard equipment. The results indicate that MA+ can be a flexible and scalable system for furthering the improvement of railway safety.
\end{abstract}

\section{Introduction}

To guarantee railway transport safety, various technologies have been applied. The latest European Train Control System (ETCS), which is based on the moving block principle, safely optimizes the maximum capacity of the rail network. Different kinds of data are submitted to the Centralized Traffic Control (CTC) system, such as the interlocking information, train position, and train diagram. Hence, CTC has a "God's view" of all trains and interlocking details [1]. The Radio Block Center (RBC) transfers the movement authority (MA) to the trains in its scope of jurisdiction. The train vehicles can only passively move based on the MA. Once the MA is a fault, it may lead to a risk scenario, which results in catastrophic consequences.

For instance, in the 2016 Berlin Tram-Crash, two commuter trains collided on a single-track stretch of railway in Germany; additionally, there was a head-on collision involving two passenger trains in southern Italy; two cargo trains collided in Finland; the 7.23 Yongwen line train collision and the Shanghai Metro Line 10 collision in China are further examples [2]. As shown in Figure 1, even though the latest technologies have been implemented, accidents happened all the same. Hence, we should not be lulled by the guarantee of the train control system safety.

Based on the technology trends, the train control system should weaken the proportion of ground faculties and provide trains with more information than in the past [3]. Some projects based on the vehicle-centric communications have been carried out in these years [4]. For instance, Alstom offered a train-centric communications-based train control (CBTC) system. This system can carry out direct train-to-train communication and control the switch by the train. With implementing this system, the maintenance costs have been decreased by $20 \%$ and energy has been saved up to $30 \%$ [5]. Publication [6] introduces a train collision avoidance system, which is based on the Global Navigation Satellite System (GNSS) to obtain the location data; potential 


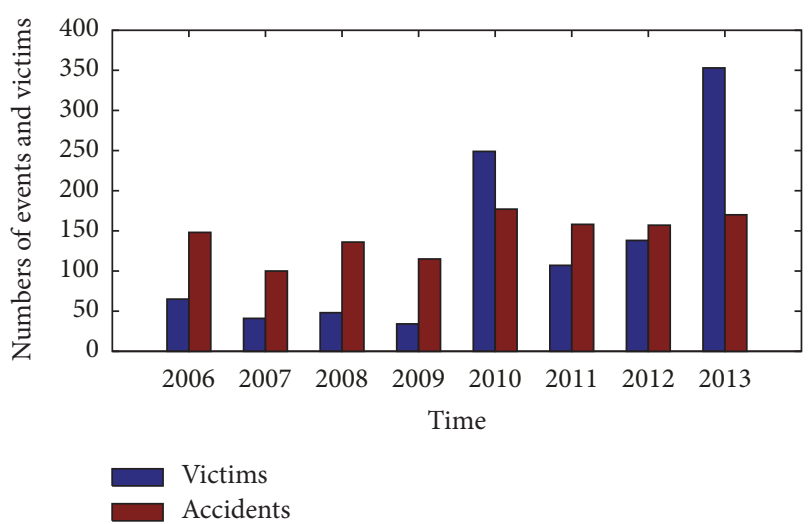

FIGURE 1: Numbers of events and victims of train collisions and derailments in Europe from 2006 to 2013.

collisions are avoided in the future. Publication [7] proposed a direct vehicle-to-vehicle distance measurement system in railways, and the system availability is validated by using colored Petri nets.

Varying from the systems mentioned in publications [57], in this paper, we propose a system that requires no additional position and speed measurement system. The essential information is collected using the internal wireless packets generated by ETCS's onboard equipment. The vehicle-tovehicle data exchange is implemented via a vehicle-centric communication link.

Improving transport safety requires numerous suitable methods. Reducing transitions to hazardous operations and increasing transitions to safer operations are the most effective implementation methods [8]. Limiting movement violation is one of the most efficient and innovative methods to reduce accidents, such as train-to-train and train-object collisions. This paper intends to provide researchers with a new movement authority (MA+), which combines advantages of the vehicle-centric communication with current movement authority (MA) mechanisms.

MA+ works as a supplementary part of the ETCS. When the ETCS is working normally and there is no potential risk, $\mathrm{MA}+$ will remain silent. Otherwise, MA+ outputs a warning or alarm. It is important to know that MA+ cannot replace the statue of the current MA. This is mostly due to the fact that the safety of MA+ cannot achieve an equivalent security level as compared with the ETCS in its infancy. Furthermore, any modifications of this current system require a substantial amount of discussion and verification. This paper focuses on such a proposal and the system description.

The remainder of the paper is organized as follows: Section 2 is dedicated to discussing the system's structure and different application scenarios. The feasibility of proposing a new movement authority, which is based on the vehiclecentric communication, is also further discussed in Section 3. In Section 4, an MA+ interface demo is introduced on the Driver Machine Interface (DMI), which can be utilized for the further simulation or practical application in the actual system. Finally, Section 5 presents the conclusion and further works.

\section{Structure and Application Scenarios of MA+}

As defined in ETCS-2, the MA contains the distance information, by which the train is authorized to move forward [9]. However, no surrounding details are available for the train. The MA+ proposed in this paper can obtain extra information by applying the vehicle-centric communication method. The extra information includes but is not limited to other trains' speed and position and switch's position and situation. In this section, the system structure, logical model, and data exchange process are presented; different implementation scenarios are then further discussed.

2.1. MA+Structure and Logical Model Description. Except for the existing infrastructures in ETCS-2, there are two main components in the structure of MA+. As shown in Figure 2, the vehicle-centric communication architecture is installed in each train, and this architecture permits internal and external data exchange. The switch announcement architecture repeatedly broadcasts the switch's location and situation.

The communication link between vehicles and switches is shown in Figure 3. In the vehicle-centric communication architecture, the $M A+$ algorithm collects the train messages from the onboard equipment and transmits the information via the Transceiver Unit. These messages provide the train's MA, speed, position, direction, and vehicle ID number. The Transceiver Unit is also in charge of receiving the train messages sent by other vehicles and switch messages from the switch announcement. After obtaining the MA and localization data, the $M A+$ algorithm matches the digital map and displays which particular track the train is on through the DMI. When there are potential hazardous scenarios, the $M A+$ algorithm will output alarms.

Before the communication link between vehicles and switches is established, MA+ works in a surveillance mode (Smod). As shown in Figure 4, there are no switches and trains in its detection range; the blue line and the yellow line represent the original MA data and the MA route, respectively. The system continuously detects the situation of potential nearby switches and trains.

The overall logical model of the MA+ algorithm is shown in Figure 5. MA+ starts from IDLE and turns into Smod after obtaining the train message data. Combining with the digital map, the MA+ shows which track the train is on $(M A+-T)$. Once the information is sufficient to be updated, the MA+ turns into corresponding modes $(\mathrm{T}, \mathrm{V}$, and $\mathrm{S}$ represent track, vehicle, and switch, resp.). For instance, $M A++_{-}{ }_{-} S$ displays the track and switch details once the MA+ obtains the information broadcasted by the switch announcement architecture. Some specific situations trigger shortening $M A$ (SMA), and the MA will be updated based on a new end of authority (EOA). In the following section, the logical details are discussed based on different scenarios, which are used to describe how $\mathrm{MA}+$ is implemented.

2.2. MA+ Implementation Scenarios. A switch can lead a train onto a different path. Hence, obtaining the position and situation of the approaching switch is essential in the MA+ implementation. Vehicle-to-switch communication provides 


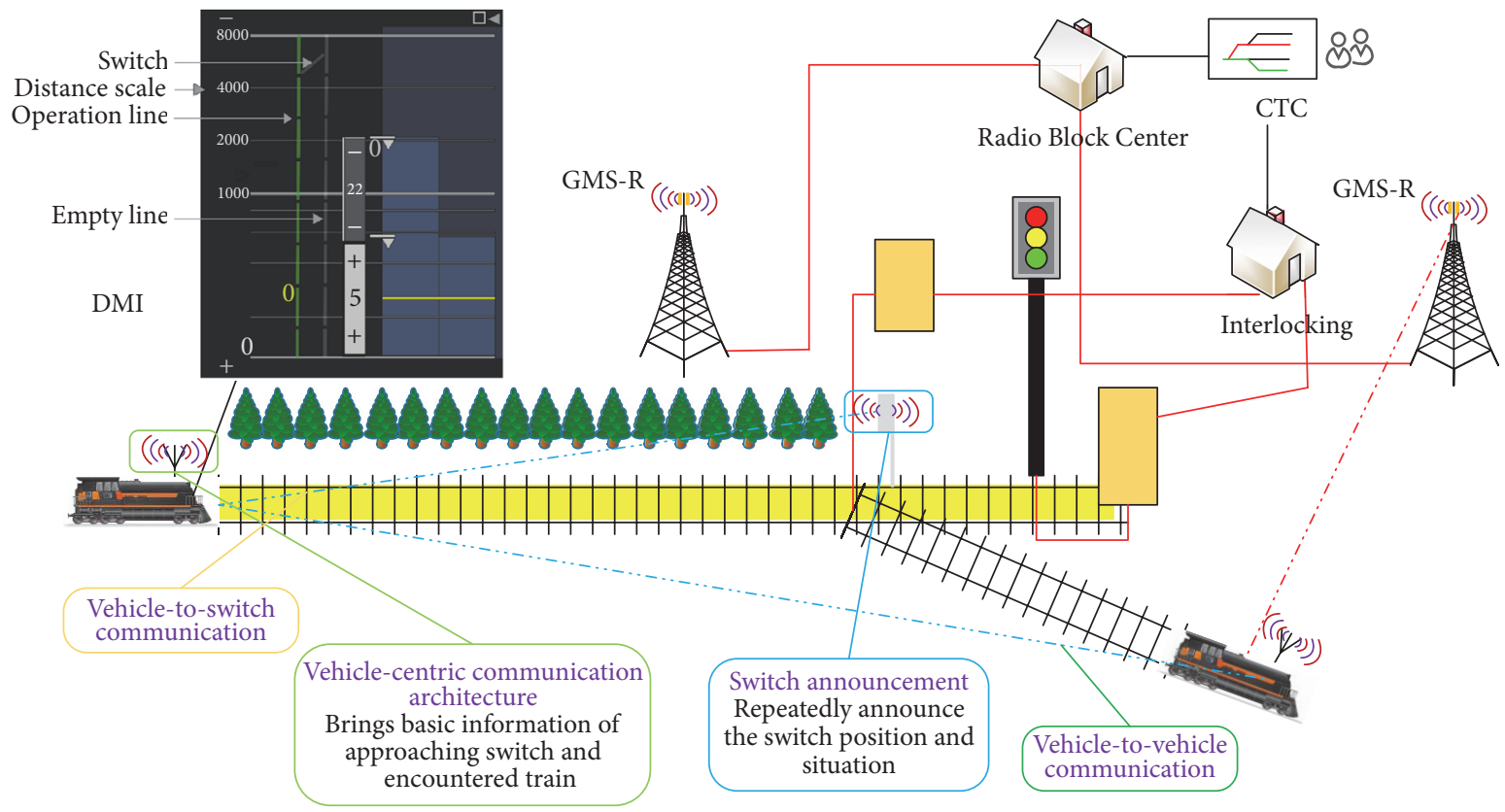

FIgURE 2: Structure of MA+.

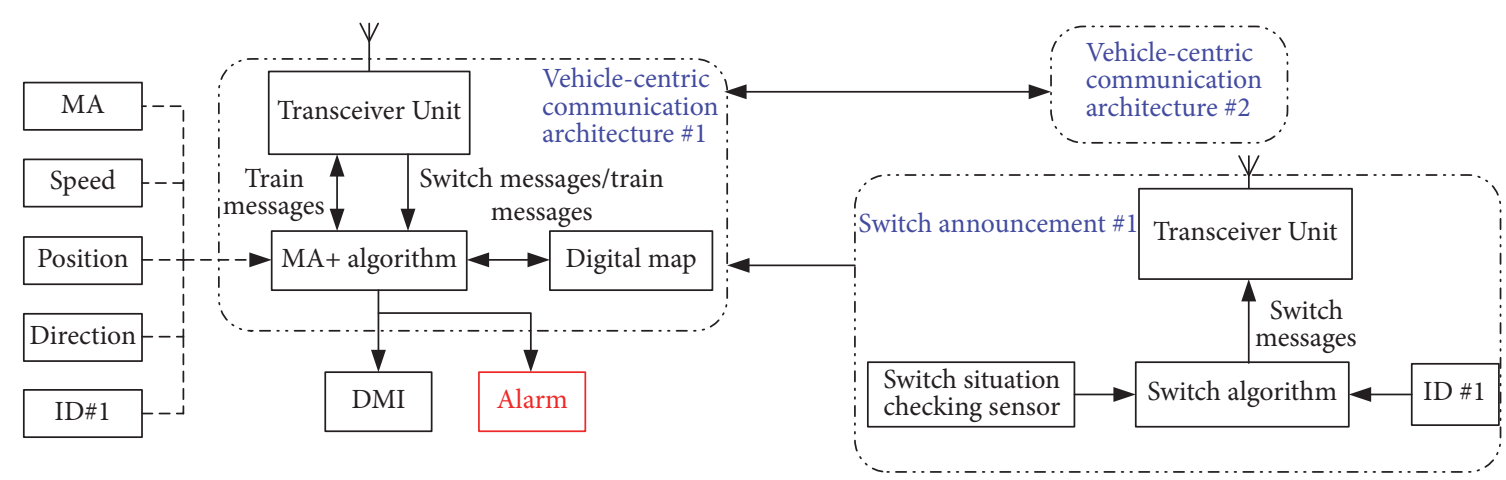

FIgURE 3: Communication link between vehicles and switches.

fundamental vector information. The following procedures are involved: surveillance, detection and appropriate avoidance, and output results:

(i) Surveillance: the local MA+ works in a monitoring mode. The switch announcement continuously broadcasts its location and position

(ii) Detection and appropriate avoidance: once the connection between the switch announcement and the local vehicle-centric communication architecture is established, the local system turns to the appropriate avoidance mode

(iii) Output results: after obtaining the position and situation of the approaching switch, the system's output results vary depending on different scenarios

In the appropriate avoidance procedure, the switch conditions are described as shown in Figure 6. In this section, four different combinations of the switch conditions are discussed to do the illustration. The red line represents the SMA. The blue and yellow rectangles indicate the normal and reverse switch positions, respectively. The switch's name turns into green or yellow depending on the switch position.

In the normal operation the switch position can be shown as Figures 6(a) and 6(b), which represent the situation that the switch is in normal and reverse positions, respectively. When the switch is in an uncertain position, it will be marked with red-dotted lines as shown in Figures 6(c) and 6(d).

It is important to note that situations 3 and 4 do not exist when the interlocking system is working correctly. The ETCS is a critical safety system, and the MA generation obeys specific fault-safety strategies. Under the scenario illustrated in Figure 6(c), switch split is likely to occur along with derailments and side collisions. Hence, among these four different scenarios, only this one can trigger the SMA. Additionally, to reduce the frequency of false alarms, the following condition is considered. When the original MA is available and the database shows that there is an approaching switch but the vehicle-to-switch connection is not established, the system ignores this scenario and no SMA is required. 


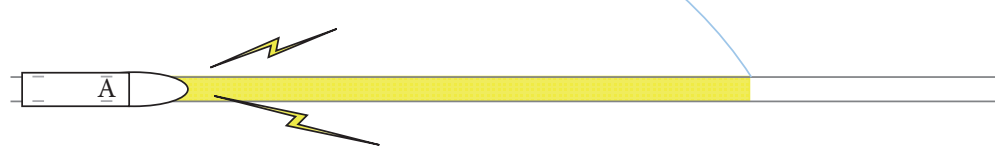

FIGURE 4: MA+ surveillance mode.

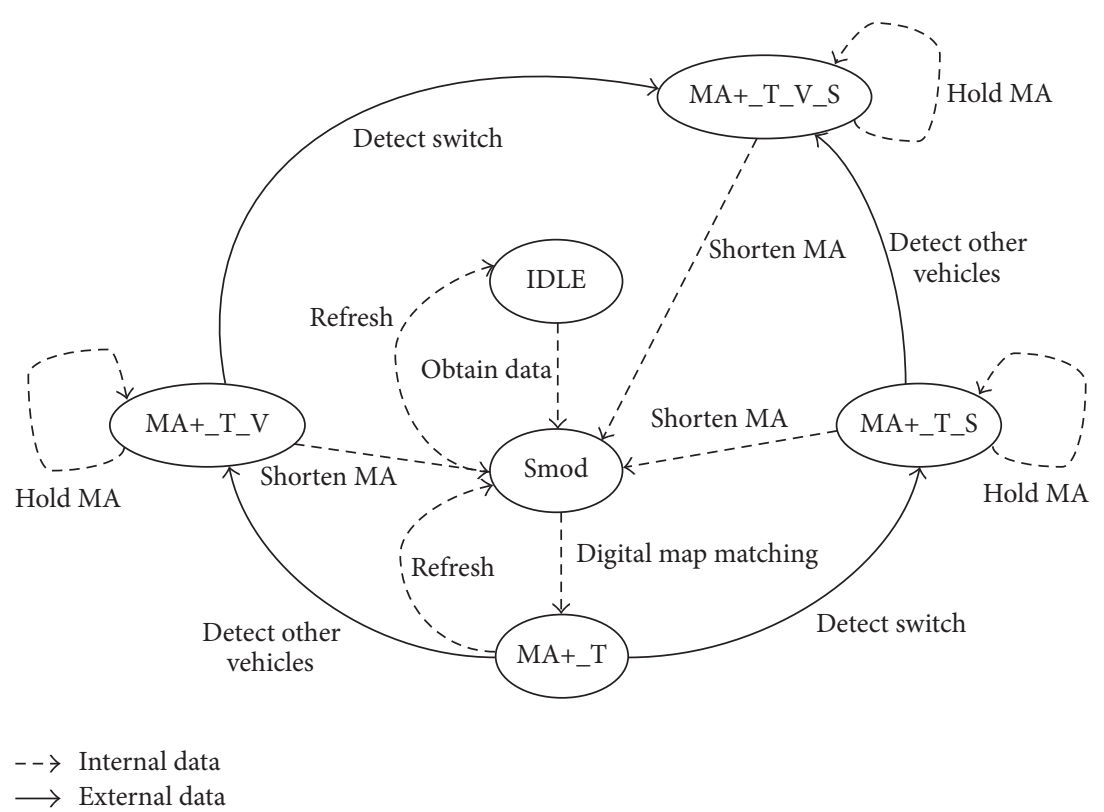

FIGURE 5: Logical model of the MA+ algorithm.

Different like the vehicle-to-switch communication, more information has to be taken into consideration when the vehicle-to-vehicle communication is established. If train $A$ detects another train $B$ in its detection range, train $A$ communicates with train $B$ and obtains the MA+ details of train $B$. In general, the scenarios can be divided into two main parts:

(i) There is no overlap of the two MA, as shown in Figure 7(a). The green part is the available extension area for the detected trains' MA. Under this scenario, train A's EOA can be reached without a risk for a hazardous situation (the train position is calculated based on the train's head position; the absolute real length of the train should be considered when SMA is triggered).

(ii) If the routes of train $A$ and $\operatorname{train} B$ have an overlap as shown in Figure 7(b), both of them have to activate the SMA based on their speed and position to prevent collisions as shown in Figure 7(c).

The practical situations are the combinations of aforementioned scenarios of switches and trains. When drivers are required to take responsibility, they can have an extended version of the surrounding switches and trains in a certain distance with the assistance of MA+. For drivers, the benefit of MA+ implementation is that it helps them understand better the surrounding environment of tasks they have to perform, especially in special scenarios where the drivers have to make sure the situation of the train ahead is safe. For instance, the onboard equipment works in modes as ON SIGHT (OS), ISOLATION (IS), and so on. Hence, MA+ is an efficient way to extend drivers' ability and improve the operational safety.

The contemporary control system has a high safety level. However, if the signal system fails, the "driver see and avoid" will be activated. MA+ can output alarm and shorten the MA automatically; furthermore, it should be kept in radio silence if there is no potential accident. Otherwise, MA+ can also be turned on manually. Hence, it is clear that MA+ will not increase drivers' responsibility and workload. Additionally, it can also provide additional communication other than just the communication between trains and ground.

\section{MA+ Detection Range Estimation}

Any theoretical proposal should be put forward in engineering implementation, and then the proposal makes sense. Before the practice using, a suitable simulation based on an existing technology is essential. For the vehiclecentric communication, different kinds of communication technologies are available, such as Global System for Mobile Communications (GSM), Code Division Multiple 


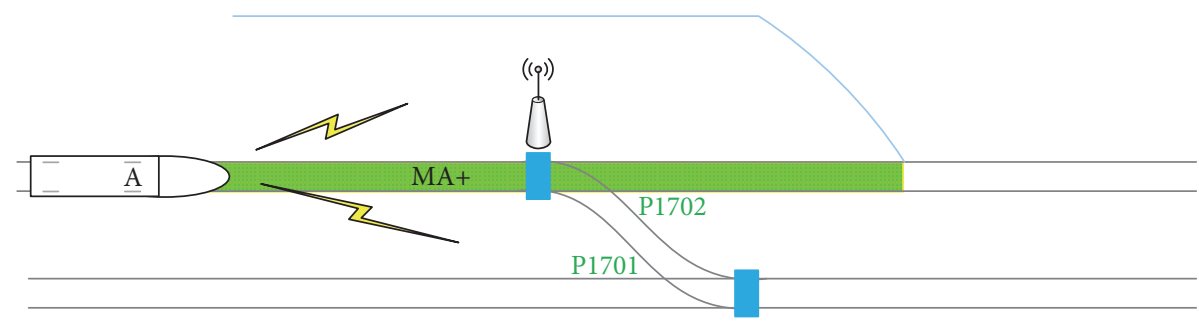

Normal position

(a)

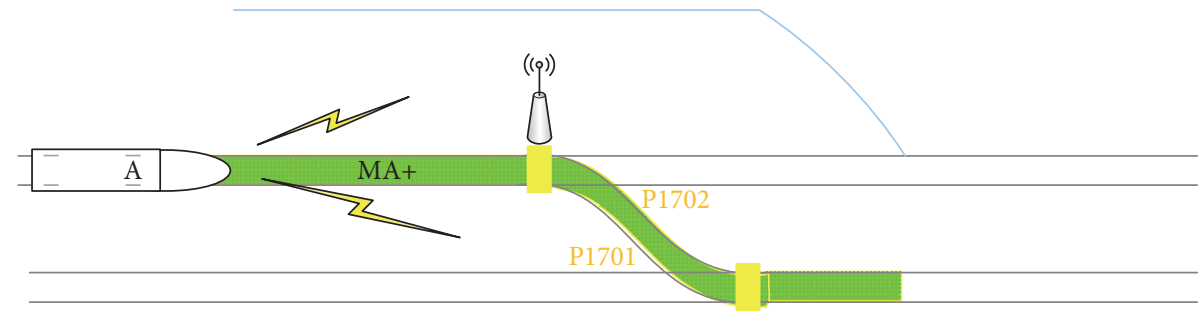

Reverse position

(b)

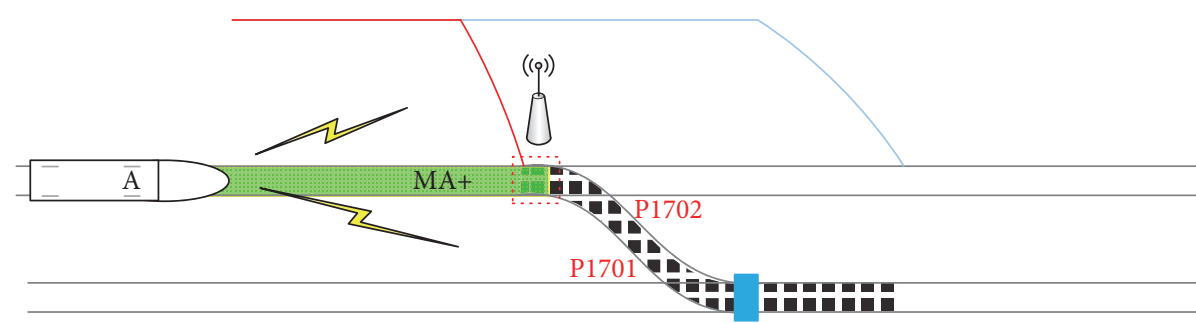

Normal position

Uncertain

(c)

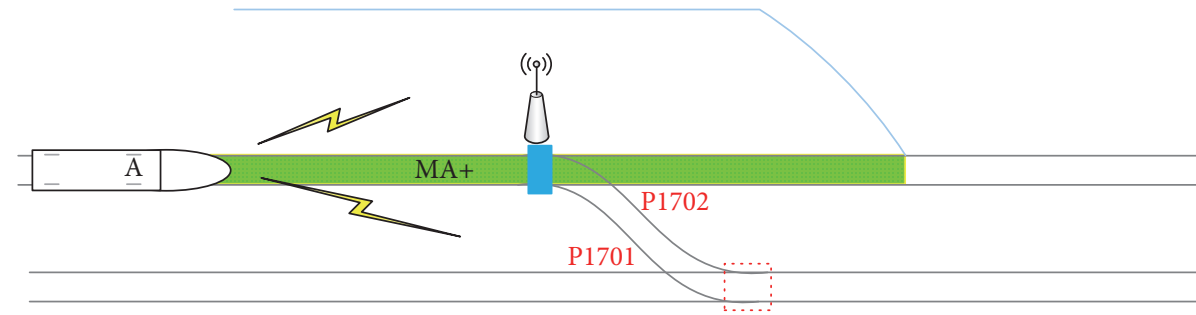

Normal position

Uncertain

(d)

FIGURE 6: MA+ operates with approaching switch scenarios.

Access (CDMA), Wideband Code Division Multiple Access (WCDMA). In recent years, the evolution of the data communication technology promotes the application of wireless communication in rail transportations, for the Long-Term Evolution (LTE) as an example [10]. Based on the high capacity and speed of LTE and less time delay (as shown in Table 1), direct communications between waysides and vehicles are possible. This will enhance the safety level of the previous control method that relied principally on the information delivered from RBC.

For the vehicle-centric communication, the detection range is an important assessment target. In this section, the 


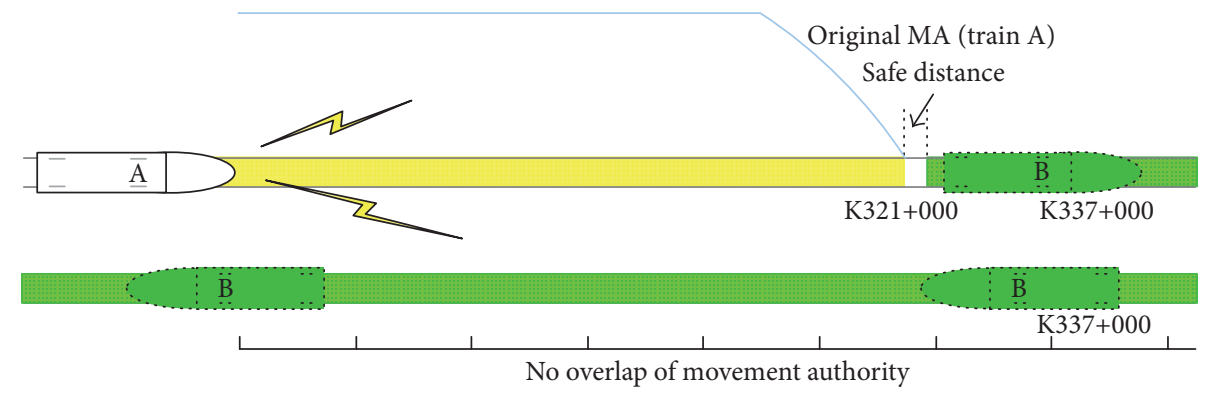

(a)

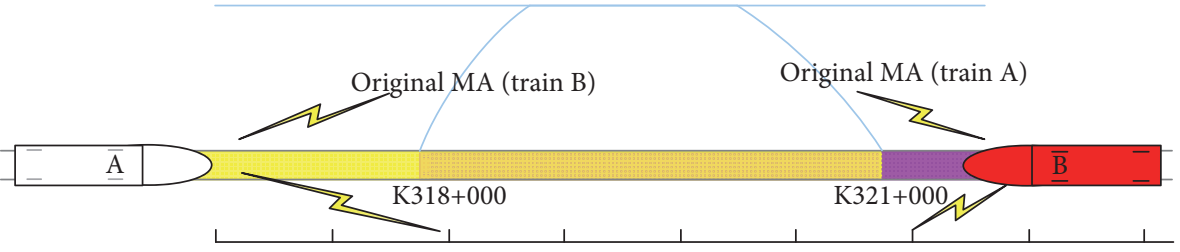

(b)

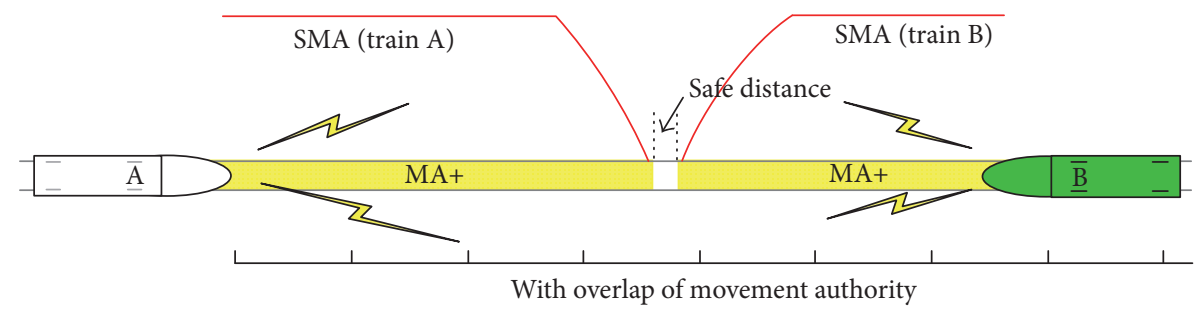

(c)

FIGURE 7: MA+ operates with encountering trains.

TABLE 1: The maximum time delays comparison of mobile communication system.

\begin{tabular}{lc}
\hline Communication system & Maximum time delay $\left(10^{-6} \mathrm{~s}\right)$ \\
\hline GSM & 1.805 \\
CDMA2000 & 0.813 \\
WCDMA & 0.130 \\
LTE & 0.030 \\
\hline
\end{tabular}

distance detection range of $\mathrm{MA}+$ is discussed based on the path loss calculation.

Different factors have various influences on the signal quality. One harsh transition environment is that in nonline-of-sight (NLOS) propagation with mountain barrier, for example, in railway curve lines, as shown in Figure 8. Under this scenario, the path loss in curve line is treated as a single round obstacle for mathematical calculations. Here,

$h$ is height of the curve line above the straight line of the local train and detected train,

$R$ is the radius of the curve line,

$d_{1}, d_{2}$ are the tangent lines through the local train and detected train position.

The diffraction loss is influenced by the frequency, curve radius, and distance. The mathematical calculation is based

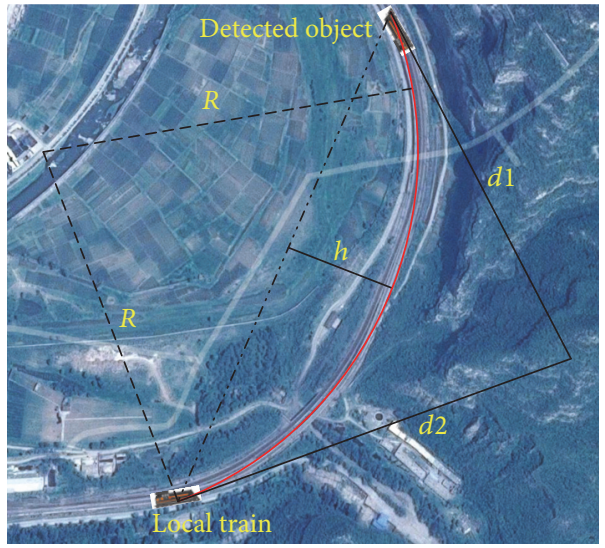

FIGURE 8: The power attenuation simulation in the curve line application scenario.

on the International Telecommunication Union (ITU) recommendation [11]. The attenuation loss $A$ can be calculated as follows:

$$
A=J(v)+T(m, n),
$$

where $J(v)$ is the Fresnel-Kirchhoff loss caused by equivalent blade shape barrier and it can be given by 


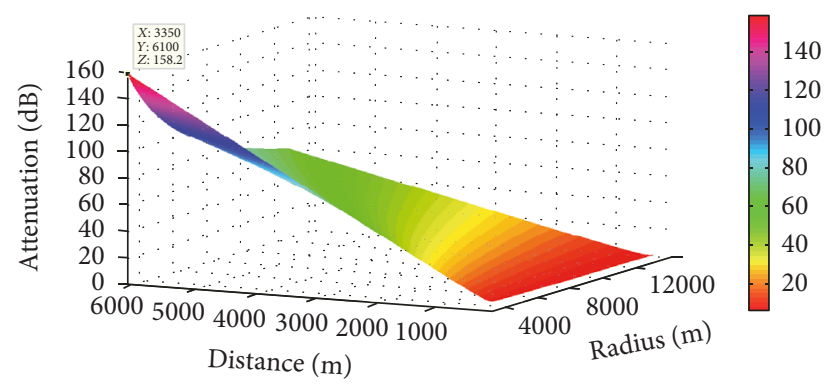

FIgURE 9: Wireless power attenuation with distance and radius.

$J(v)$

$$
=-20 \log \left(\frac{\sqrt{[1-C(v)-S(v)]^{2}+[C(v)-S(v)]^{2}}}{2}\right),
$$

where $C(v)$ and $S(v)$ are the real and imaginary parts of Fresnel integral, respectively. For the transition with a barrier blocking the line-of-sight transmission, $J(v)$ can be approximately described as follows:

$$
\begin{gathered}
J(v)=6.9+20 \log \left(\sqrt{(v-0.1)^{2}+1}+v-0.1\right), \\
v=0.0316 h\left[\frac{2\left(d_{1}+d_{2}\right)}{\lambda d_{1} d_{2}}\right]^{1 / 2},
\end{gathered}
$$

where $h$ and $\lambda$ are in meters, $\lambda$ is the wavelength, and $d_{1}, d_{2}$ are in kilometers.

$T(m, n)$ is the additional loss caused by barrier curvature, which is the curve line radius $R . m$ and $n$ are given by (5) and (6), respectively:

$$
\begin{aligned}
& m=\frac{R\left[\left(d_{1}+d_{2}\right) / d_{1} d_{2}\right]}{[\pi R / \lambda]^{1 / 3}}, \\
& n=\frac{h[\pi R / \lambda]^{2 / 3}}{R} .
\end{aligned}
$$

When $m n \leq 4, T(m, n)$ equals (7); when $m n>4, T(m, n)$ equals (8).

$$
\begin{aligned}
T(m, n)= & 7.2 m^{1 / 2}-(2-12.5 n) m+3.6 m^{3 / 2}-0.8 m^{2} \\
T(m, n)= & -6-20 \log (m n)+7.2 m^{1 / 2}-(2-17 n) m \\
& +3.6 m^{3 / 2}-0.8 m^{2}
\end{aligned}
$$

In Europe, LTE frequencies are bands 1/3/7/8/20. Band 8 is currently used mostly by GSM. Band 8 is attractive from a coverage point of view due to the lower propagation losses. The band can be reused for LTE or HSPA. Bands 8 and 20 hold the uplink frequencies $880-915 \mathrm{MHz}$ and $832-862 \mathrm{MHz}$. The downlink frequencies are $925-960 \mathrm{MHz}$ and $791-821 \mathrm{MHz}$ [12]. Here we choose the frequency $930 \mathrm{MHz}$ to do the simulation. The result is shown in Figure 9, which indicates

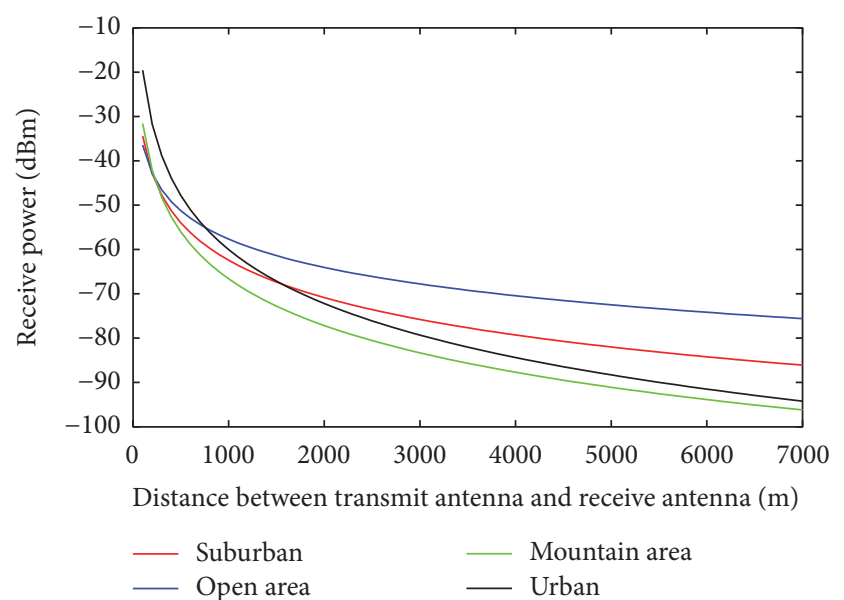

FIGURE 10: Receive signal power in different scenarios.

the interrelationship among the diffraction loss, detection distance, frequency, and radius. The received signal power should be greater than the sensitivity of a receiver, as shown in (9).

$$
\begin{aligned}
P_{r}(d)[\mathrm{dBm}] & =P_{t}[\mathrm{dBm}]+G-A-P L_{\Delta}, \\
G & =10 \log \left(G_{t} G_{r}\right),
\end{aligned}
$$

where $P_{r}(d)[\mathrm{dBm}]$ is the received power; $P_{t}[\mathrm{dBm}]$ is the transfer power; $G$ is the gain; $G_{t}$ and $G_{r}$ are the gains of the transfer and receiver antennas, respectively; $P L_{\Delta}$ is the attenuation caused by device and feeder cable.

For the received power, some experimental data is available to be referenced. In publication [13], the path loss measurements at the $930 \mathrm{MHz}$ in the different scenario were done. The empirical power of received signal models for suburban area, open area, mountain area, and urban area was proposed, as shown in (11), (12), (13), and (14), respectively. The simulation result is shown in Figure 10. As shown in the result, the received signal power is greater than $-90 \mathrm{dBm}$ within $4000 \mathrm{~m}$. Hence, the detection range is not a limitation of this system's practical application. Taking ICE-3 as an example, the train emergency braking distance is between 2300 and $2800 \mathrm{~m}$ depending on the actual speed.

$$
\begin{aligned}
& P_{r}(d)=21.577-28.001 \log (d), \\
& P_{r}(d)=6.0246-21.2261 \log (d), \\
& P_{r}(d)=38.432-35.015 \log (d), \\
& P_{r}(d)=61.337-40.452 \log (d) .
\end{aligned}
$$

Here we take an actual line as a case study. The minimum railway curve radius is different in various railway lines, and several cases are shown in Table 2 [14]. The simulation results indicate that, under the minimum curve radius of $3350 \mathrm{~m}$ in Köln-Rhein/Mann line, the signal attenuation is $158.2 \mathrm{~dB}$ when the detected distance is $6100 \mathrm{~m}$. In current practice application, the typical maximum path loss of LTE can be $163.5 \mathrm{~dB}$ [15]. In order to make the detection range as further 
TABLE 2: Symbol form/shape and descriptions.

\begin{tabular}{lc}
\hline Symbol No. & Description \\
\hline SW1 & Switch in normal position \\
SW2 & Switch in reverse position \\
SW3 & Switch in reverse and normal position \\
SW4 & Switch in normal and reverse position \\
TR1 & \\
TR2 & Train without collision risk \\
TC1 & Train with collision risk \\
TC2 & Operation line \\
\hline
\end{tabular}

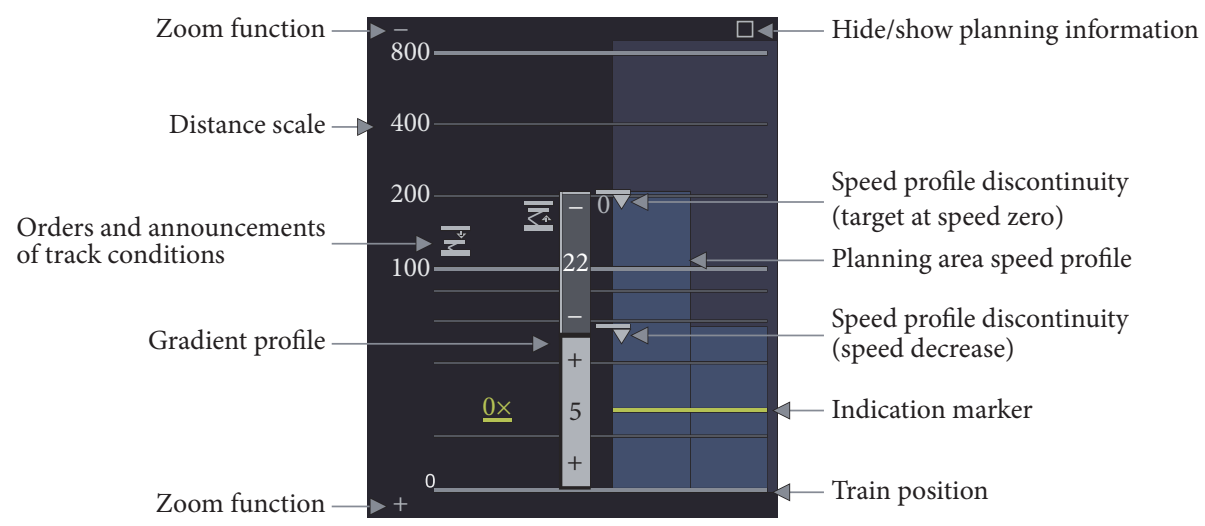

FIGURE 11: Main objects of the planning information.

as possible, many different methods can be used, for instance, choosing a suitable wireless frequency spectrum according to the simulation result in Figure 9, increasing transmitter power, enhancing receiver sensitivity, and building repeater stations. Both the simulation result and practical results indicate that the vehicle-centric communication is available at the technical level. Hence, the detection range is not a limitation of the MA+ practical application.

\section{An Application Demo on the DMI}

The MA+ can be merged into the current train control system. In this section, a demo is proposed on the DMI of ETCS. The onboard equipment displays the essential information on the DMI for drivers. MA+ is combined with the planning information on DMI. On DMI, the orders and announcements overview displays within the MA and up to the first target at zero speed. The following aspects are involved, if any, as shown in Figure 11.

Here we add MA+ information in orders and announcements of track conditions area, and the basic symbols are shown in Table 3. Symbols or shapes having a certain meaning in general railway control systems are avoided, and the display permits no interaction with the driver during the normal operation.

Operation line and empty line are defined in the local train's view. Based on the switch conditions, there are different MA operation routes. The driver will know which route is being implemented. As shown in Figure 12, the length of MA is assumed to be $800 \mathrm{~m}$. The train was transferred from the current operation line to the empty line through SW2, which locates at $280 \mathrm{~m}$ in front, as shown in Figure 12(b). SW3 and SW4 are in hazardous conditions; trailed switch accidents may happen. SW3 will lead the local train to another track and switch split will occur. Hence, SMA is required and an alert is triggered. There is no risk of collisions to the local train under SW4, and no SMA action can be triggered automatically.

Once other trains are detected and if there is no overlap between operation lines, the system will show the positions of other trains as green symbols. The angle shows the train operation direction. If there are overlaps between two operation lines, SMA is immediately executed. Then, because of different train directions, two different scenarios should be discussed: scenario one: after the new EOA was refreshed, there is still an overlap of the MA of two trains; both two trains have to shorten their MA again based on their speed, distance, and location, as shown in Figure 13(a); scenario two: if the detected trains and local train have the same route direction, the new EOA will be updated based on the end of the detected train, as shown in Figure 13(b).

\section{Conclusion and Further Work}

In this paper, a new movement authority based on vehiclecentric communication was proposed to increase the safety level of the railway operation. The structure of the system was given, and implementation scenarios were also discussed. 
TABLE 3: Comparison among different curve radiuses.

\begin{tabular}{|c|c|c|c|c|c|c|c|}
\hline Organization & JR & JR & DB & $\mathrm{DB}$ & SNC & SNCF & $\mathrm{CRH}$ \\
\hline Item & Tokaido Shinkansen & Tokyo-Joetsu & Hannover-Wrzburg & Köln-Rhein/Mann & Paris-Sud-Est & Atlantique & Beijing-Shanghai \\
\hline $\begin{array}{l}\text { Maximum } \\
\text { design speed } \\
\mathrm{km} / \mathrm{h}\end{array}$ & null & 280 & 300 & 300 & 350 & 380 & 380 \\
\hline $\begin{array}{l}\text { Maximum } \\
\text { service speed } \\
\mathrm{km} / \mathrm{h}\end{array}$ & 300 & 275 & 250 & null & 270 & 300 & 300 \\
\hline $\begin{array}{l}\text { Minimum curve } \\
\text { radius } m\end{array}$ & 4000 & 4000 & 7000 & 3350 & 4000 & 6250 & 7000 \\
\hline
\end{tabular}

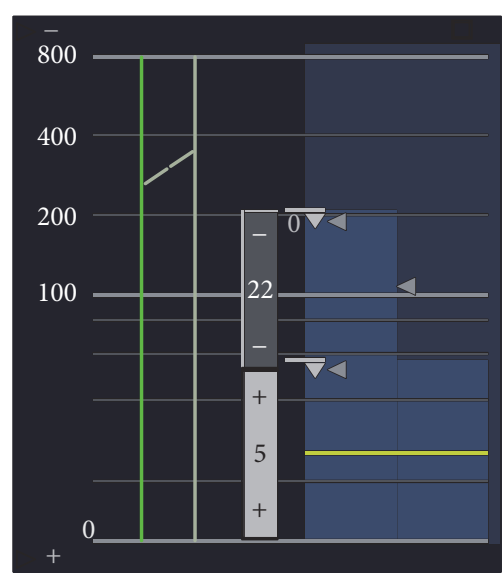

(a)

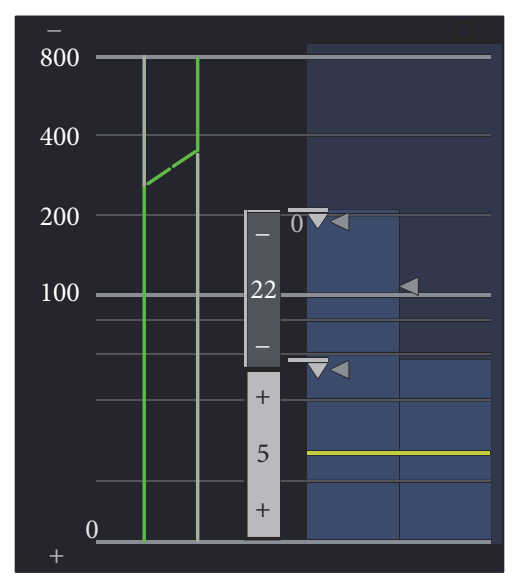

(b)

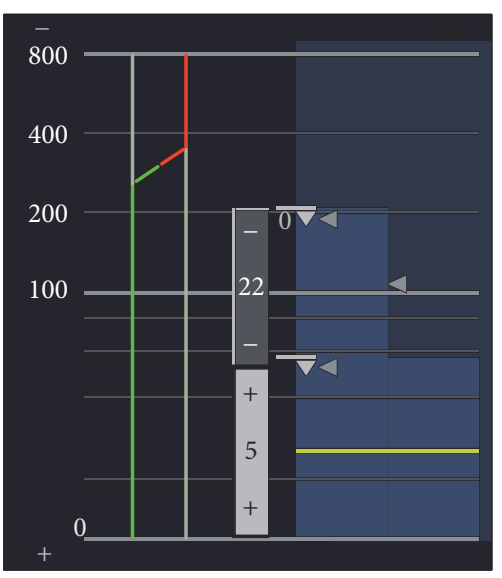

(c)

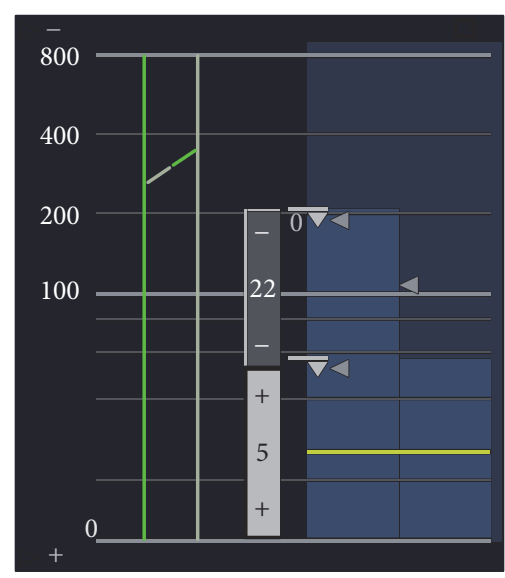

(d)

FIGURE 12: DMI operates with approaching switch scenarios.

With the assistance of vehicle-centric wireless communication, local trains can have an overview of surrounding scenarios. By estimating the detection range based on attenuation calculation, the results indicated that this vehicle-centric communication proposal was engineering-feasible. Finally, a demo for the MA+ application on DMI was presented. It is clear that similar vehicle-centric technologies can be widely used in the future to increase the safety level of railway transport.
In further works, an MA+ prototype machine will be designed, which can carry out the fundamental functions proposed in this paper. Given that the current DMI has been developed with a view to optimizing information provision and providing drivers with the information they need, any change to the DMI and information provided should be validated under careful consideration. Hence, in the following research, we will try to get railway companies support to revise the system's industry requirements and provide a more 


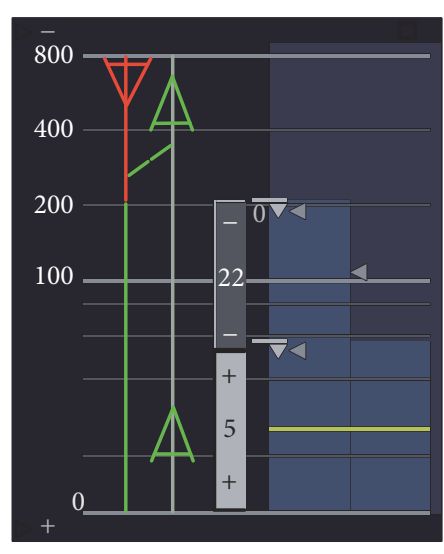

(a)

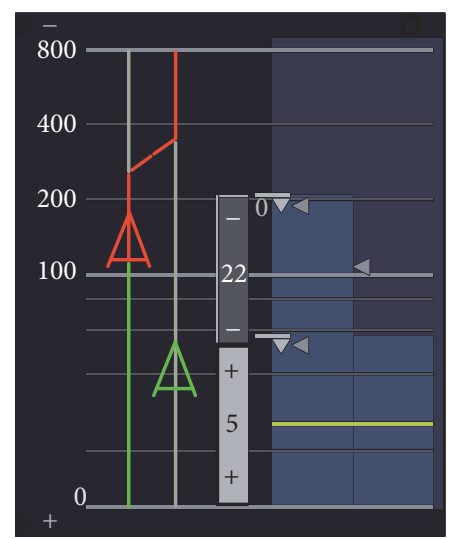

(b)

FIgURE 13: DMI with detected trains.

actual analysis. A full function device will be available to be applied on the actual DMI.

\section{Conflicts of Interest}

The authors declare that there are no conflicts of interest regarding the publication of this paper.

\section{Acknowledgments}

This work is supported by the National Natural Science Foundation of China (U1734211 and 61603026), Shanghai Committee of Science and Technology research program (17DZ1100106), and Beijing Municipal Natural Science Foundation (I18E300010 and L171004).

\section{References}

[1] H. Song and E. Schnieder, "Modeling of railway system maintenance and availability by means of colored petri nets," Maintenance and Reliability, vol. 20, no. 2, pp. 232-239, 2018.

[2] H. Dong, B. Ning, Y. Chen et al., "Emergency management of urban rail transportation based on parallel systems," IEEE Transactions on Intelligent Transportation Systems, vol. 14, no. 2, pp. 627-636, 2013.

[3] J. Wang, J. Wang, C. Roberts, and L. Chen, "Parallel monitoring for the next generation of train control systems," IEEE Transactions on Intelligent Transportation Systems, vol. 16, no. 1, pp. 330-338, 2015.

[4] B. Ai, X. Cheng, T. Kurner et al., "Challenges toward wireless communications for high-speed railway," IEEE Transactions on Intelligent Transportation Systems, vol. 15, no. 5, pp. 2143-2158, 2014.

[5] J. Moreno, J. Riera, L. De Haro, and C. Rodriguez, "A survey on future railway radio communications services: challenges and opportunities," IEEE Communications Magazine, vol. 53, no. 10, pp. 62-68, 2015.

[6] A. Lehner, T. Strang, and C. R. Garca, "A reliable surveillance strategy for an autonomous rail collision avoidance system," in Proceedings of the 15th ITS World Congress, New York, NY, USA, 2008.
[7] H. Song and E. Schnieder, "Validation, verification and evaluation of a train to train distance measurement system by means of colored petri nets," Reliability Engineering and System Safety, vol. 164, pp. 10-23, 2017.

[8] E. Schnider, "Traffic safety and availability - contradiction or attraction," in Proceedings of 2nd International Symposium of Networks for Mobility, 2004.

[9] "Ertms/etcs - baseline 3, system requirements specification. chapter 3, principles, subset-026-3, issue 3.0.0. 23, 2008".

[10] L. Lei, J. Lu, Y. Jiang et al., "Stochastic delay analysis for train control services in next-generation high-speed railway communications system," IEEE Transactions on Intelligent Transportation Systems, vol. 17, no. 1, pp. 48-64, 2016.

[11] ITU-R Recommendation P.526-11, "Propagation by diffraction, 2009".

[12] H. Holma and A. Toskala, LTE for UMTS: Evolution to LTEadvanced, John Wiley and Sons, 2011.

[13] R. He, B. Ai, Z. Zhong, A. F. Molisch, R. Chen, and Y. Yang, "A measurement-based stochastic model for high-speed railway channels," IEEE Transactions on Intelligent Transportation Systems, vol. 16, no. 3, pp. 1120-1135, 2015.

[14] M. Lindahl, "Track geometry for high-speed railways," TRITAFKT Report, Tech. Rep., 54.

[15] H. Abid, T. C. Chung, S. Lee, and S. Qaisar, "Performance analysis of lte smartphones-based vehicle-to-infrastrcuture communication," in Ubiquitous Intelligence and Computing and 9th International Conference on Autonomic and Trusted Computing (UIC/ATC), pp. 72-78, 2012. 


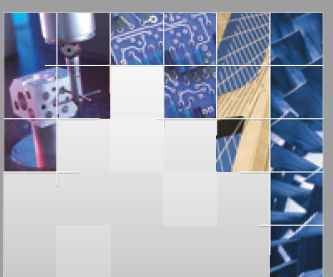

\section{Enfincering}
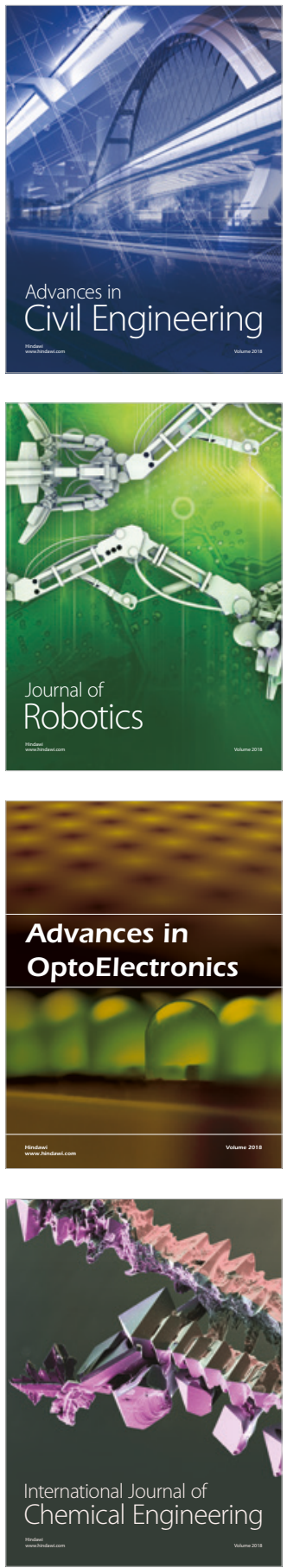

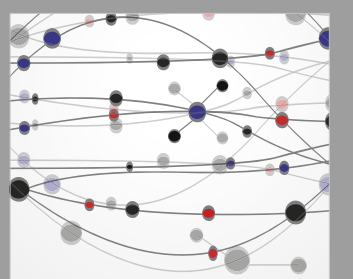

\section{Rotating \\ Machinery}

The Scientific World Journal

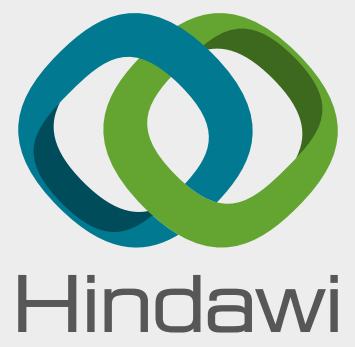

Submit your manuscripts at

www.hindawi.com
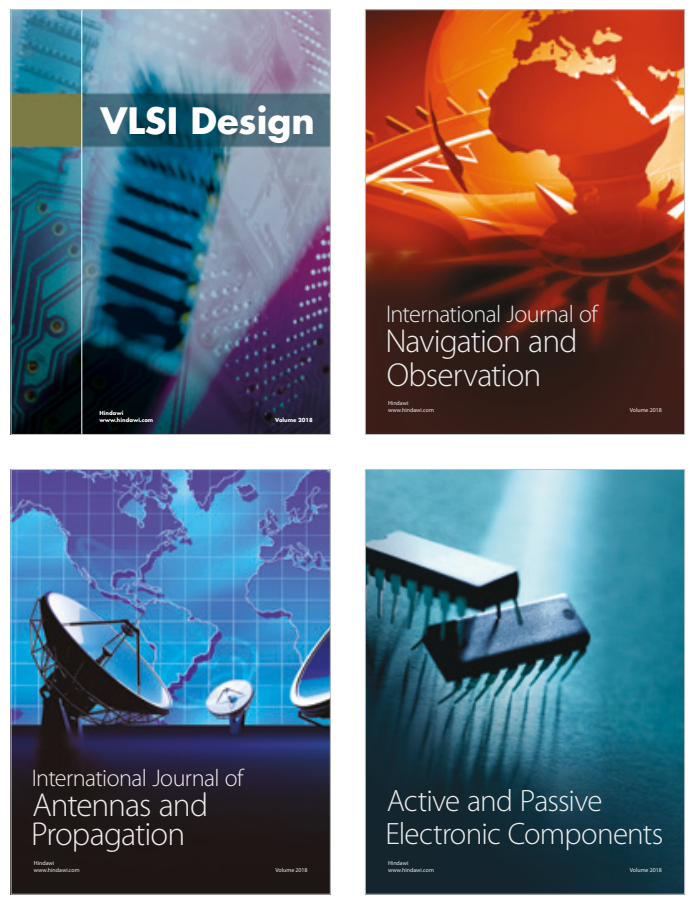
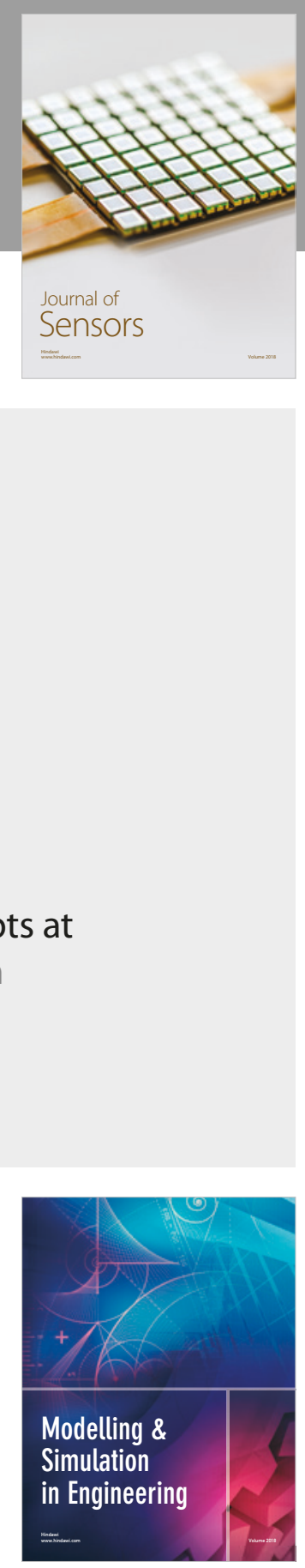

\section{Advances \\ Multimedia}
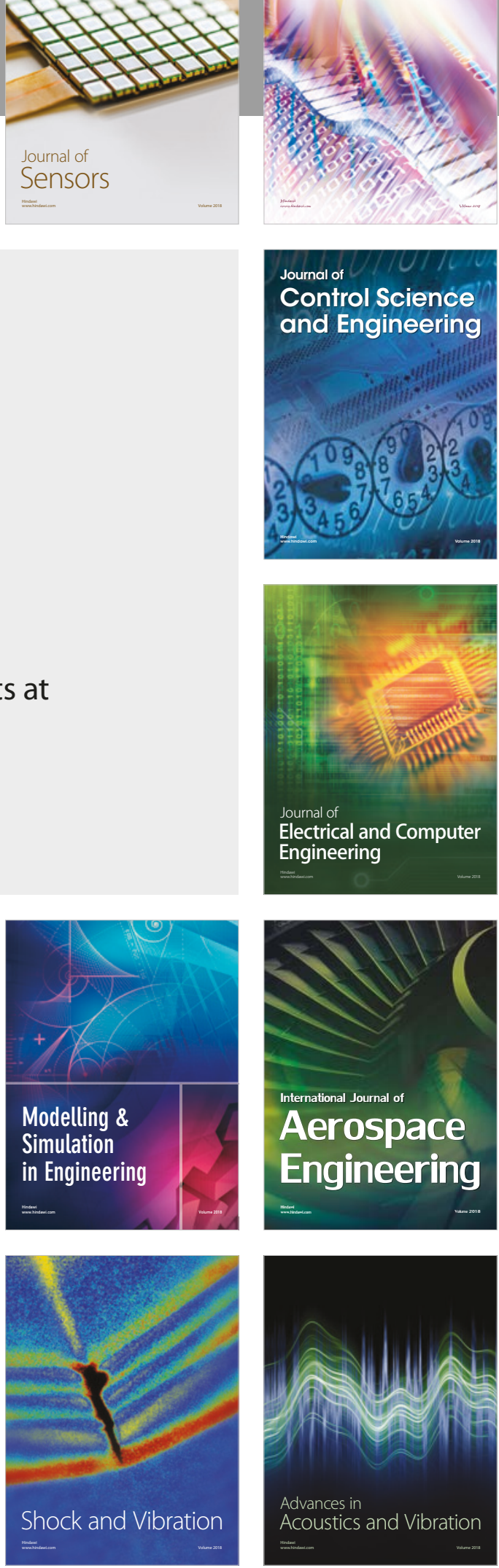